\title{
FINITE SIZE EFFECTS FOR THE DILUTE COUPLING DERRIDA MODEL
}

\author{
large Allakhverdyan A.E., Saakian D.B. \\ Yerevan Physics Institute \\ Alikhanian Brothers St.2, Yerevan 375036, Armenia \\ Saakian@ @x1.YERPHI.AM
}

\begin{abstract}
We consider paramagnetic, spin-glass and ferromagnetic phases. At $T=0$ model gives for the some values of connectivity (near the critical) extremal suppression of finite size effects (decoding error probability).
\end{abstract}

1. Introduction Derridas's model [1] is the simplest among the spin-glasses. It has solved in the first level of replica symmetry breaking [2].

May be this is the origin of unique feature-condition for the appearance of ferromagnetic phase coincides with the constraint of Shannon for the optimal coding.

This hypothesis [3] have proved for the general case in [4], [5]

The physical meaning of codes in the approach of Sourlas is this:

The $N$ numbers $\xi_{i}$ are given, taking values \pm 1 .

By means of them we construct $Z$ numbers $\tau_{i}(Z \geq N)$, then formulate some function of $N$ discrete variables $\sigma_{i}$

$$
H\left(\sigma_{i}, \tau_{j}\right)
$$

which has as constants $Z$ couplings $\tau_{j}$,

There is a restriction, that single global minimum is configuration

$$
\sigma_{i}=\xi_{i}
$$

It is a simple to construct such function, but then in our problem appears noise. Our

couplings $\tau_{i}$ with probability $\frac{1+m}{2}$ conserve their sign, and with probability $\frac{1-m}{2}$ change 
it. Again there is a restriction, that configuration (2) still is a single vacuum for the noisy couplings $\tau_{i}$.

It is possible to do this procedure only for the some (good!) choice of function $H$. For this case information theory gives restriction [6]

$$
Z[\ln 2-h(m)] \geq N \ln 2
$$

where for the entropy we have

$$
h(m)=-\frac{1+m}{2} \ln \frac{1+m}{2}-\frac{1-m}{2} \ln \frac{1-m}{2}
$$

Really our condition (single vacuum) is correct not with probability 1 , but a little less:

$$
1-a \exp [-E(m, N / Z) Z]
$$

The second member is just the decoding error probability.

On the language of statistical physics we will investigate magnification at $T=0$

$$
<\xi_{i} \sigma_{j}>
$$

This expression gives probability, that our condition is fulo fild. So decoding error probability is equivalent to finite size effect in the expression of magnetization

In such manner we have coded our original information- $N$ numbers $\xi_{i}$, to noisy number $\tau_{j}$. We can rederive original values of $N$ numbers $\xi_{i}$, searching (by Monte-Karlo) minimum of function $H\left(\sigma_{i}, \tau_{j}\right)$,

We want extremely suppress decoding error probability.

The information theory gives restriction for the maximal value of function $E(m, R)$, where

$$
R=N / Z
$$

is a rate of information transmission.

On the [7] finite size effects have calculation at $T=0$ for the full connectively case in Derrida model, which corresponds to limit

$$
Z / N \rightarrow \infty \quad m \rightarrow 1 / 2
$$


On the [8] has investigated fine structure of ferromagnetic phase, and has found some subphases, which differs each-others by finite size effects.

In this work we are going to calculate finite size effects for paramagnetic, spin-glass and ferromagnetic phase. For that purposes we are using methods of [9].

\section{Derivation of REM with weak connectivity}

Let us consider hamiltonian

$$
H=-\sum_{\left(i_{1} \cdots i_{p}\right)} \tau_{i_{1} \cdots i_{p}} \sigma_{i_{1}} \cdots \sigma_{i_{p}}
$$

where spins $\tau_{i}$, are quenched couplings $\tau_{i}$, taking values \pm 1 ,

In our hamiltonian there are $C_{N}^{P}$ different choice of $\left(i_{1} \cdots i_{p}\right)$ and consequently $C_{N}^{P}$ different couplings $\tau_{i_{1} \cdots i_{p}}$. We consider restriction

$$
\sum_{\left(i_{1} \cdots i_{p}\right)}\left(\tau_{i_{i} \cdots i_{p}}\right)^{2}=\alpha N
$$

In the bounds of this restriction, which means an weak correlation, all $\tau_{j}$ are distributed independently, with the same probabilities

$$
\begin{aligned}
& \tau_{i_{1} \cdots i_{p}}=0 \quad \text { with probability } \quad 1-\alpha N / C_{N}^{P} \\
& \tau_{i_{1} \cdots i_{p}}= \pm 1 \quad \text { with probability } \quad \frac{1 \pm m}{2} \frac{\alpha N}{C_{N}^{P}}
\end{aligned}
$$

On [9] authors considered only conditions (11),(12). Our restriction (10) simplifies calculations. As a results finite size effects are changed only in paramagnetic phase

Let us consider, as in [9], common distribution of $M$ energy levels.

We have $M$ configurations with the values of spins $\sigma_{i}^{\alpha}, \leq \alpha \leq M$ and values of energy $E_{\alpha}$

Let as define

$$
P\left(E_{1} \cdots E_{M}\right)=<\delta\left(E_{1}-H\left(\sigma_{i}^{1}\right)\right) \cdots \delta\left(E_{M}-H\left(\sigma_{i}^{M}\right)\right)>
$$


If it would be possible to calculate. Case with

$$
M=2^{N}
$$

Then we could calculate

$$
<\ln Z(\tau, T)>\int \prod_{\alpha=1}^{2^{N}} d E_{\alpha} P\left(E_{1} \cdots E_{2^{N}}\right) \ln \sum_{\alpha=1}^{2^{N}} \exp \left(-B E_{\alpha}\right)
$$

We have derived (15), using the fact that our system has only $2^{N}$ levels of energy.

That's why we have considered $Z$ as a function of energy levels $E_{\alpha}$ (instead of-as a function of couplings $\tau$ ). Then, due to our choice (10)-(12), $P\left(E_{1} \cdots E_{M}\right)$ factorizates.

Using Fourier representation for $\delta$ function

$$
P\left(E_{1} \cdots E_{M}\right)=\int_{-i \infty}^{i \infty} \prod_{\alpha=1}^{2^{N}} \frac{d E_{\alpha}}{2 \pi} \exp \left[\sum_{\alpha=1}^{M} E_{\alpha} \hat{E}_{\alpha}-\sum_{\alpha} \hat{E}_{\alpha} \sum_{i_{1} \cdots i_{p}} \tau_{i_{1} \cdots i_{p}} \sigma_{i_{1}}^{\alpha} \cdots \sigma_{i_{p}}^{\alpha}\right]
$$

Let us use standard representation

$$
\exp (a \sigma)=\cosh (a)[1+\tanh (a) \sigma]
$$

where $\sigma= \pm 1$.

We can derive, expanding

$$
\prod_{i_{1} \cdots i_{p}}\left[\cosh \left(\hat{E}_{1}\right)+m \sinh \left(\hat{E}_{1}\right)\right] \prod_{\alpha=2}^{M} \cosh \left(\hat{E}_{\alpha}\right)\left[1+\tanh \left(\hat{E}_{\alpha}\right) \sigma_{i_{1}}^{\alpha} \cdots \sigma_{i_{p}}^{\alpha}\right]
$$

the expression for the $P\left(E_{1} \cdots E_{M}\right)$

$P\left(E_{1} \cdots E_{M}\right)=\int_{-i \infty}^{i \infty}\left\{\left[\cosh \left(\hat{E}_{1}\right)+m \sinh \left(\hat{E}_{1}\right)\right] \cosh \left(E_{1}\right) \cdots \cosh \left(E_{M}\right)\right\}^{\alpha N} \prod_{\alpha=1}^{M} \frac{d E_{\alpha}}{2 \pi} e^{\sum_{\alpha} E_{\alpha} \hat{E}_{\alpha}}$

in the expression we neglect by effects of order

$$
\sum_{1 \leq i_{1} \cdots<i_{p} \leq N}\left(\sigma_{i_{1}}^{\alpha} \sigma_{i_{p}}^{B}\right) \cdots\left(\sigma_{i_{1}}^{\alpha} \sigma_{i_{p}}^{B}\right) / C_{N}^{P}
$$

We can consider this expression as a scalar product between 2 configurations).

Let us calculate (20) for the case, when only 1 turned spin. it is ease to derive

$$
\left(C_{N-1}^{P}-C_{N-1}^{P-1}\right) / C_{N}^{P}=\frac{N-2 P}{N}
$$


We see, that corrections disappear for the choice

$$
P=\frac{N}{2}
$$

When the number of turned spins is $\delta k$, we have expression like

$$
\frac{1}{N} e^{-C \delta N}
$$

In future calculations we using expression (19) for $P\left(E_{1} \ldots E_{M}\right)$. Then accuracy of one expression is polynomial or exponential (by $N$ ). We call this corrections as "finite $P$ corrections". Their investigation is a very hard work.

In the next section we calculating finite size effects for the model (15),(19) using the formula from [1]

$$
<\ln Z>=\Gamma^{\prime}(1)-\int_{-\infty}^{\infty} \ln t e^{-\phi} d \phi
$$

where

$$
e^{-\phi}=<e^{-t Z}>
$$

It is easy to derive, taking $Z=e^{A}$, equation

$$
A=\Gamma^{\prime}(1)-\int_{\infty}^{-\infty} u d\left[e^{-e^{U+A}}\right]
$$

\section{Pharamagnetic Phase}

In this phase, as well as in the spin-glass case, we taking $m=0$.

It is easy to derive

$$
e^{-\phi}=\left[\int_{-i \infty}^{i \infty} \frac{d E_{1} d E_{2}}{2 \pi} e^{-E_{1} E_{2}+\alpha N \ln \cosh E_{1}-t e^{-B E_{2}}}\right]^{2^{N}}
$$

Let us make transformation

$$
x=e^{-B E_{2}}, \quad E_{1}=B E
$$


After this easy to derive

$$
\begin{aligned}
e^{-\phi} & =\left[\int_{-i \infty}^{-i \infty} \frac{d E}{2 \pi} \int_{0}^{\infty} e^{-x+(E-1) \ln x-U E+\alpha N \ln \cosh (B E)}\right]^{2^{N}} \\
& =\left[\int_{-i \infty}^{-i \infty} \frac{d E}{2 \pi} \Gamma(E) e^{-U E+\alpha N \ln \cosh (B E)}\right]^{2^{N}}
\end{aligned}
$$

In this integral integration loop passed pole zero from right side.

For the integration we must lift integration loop righter, until saddle point, defined by equation

$$
\tanh (B E)=\frac{U}{\alpha N B}
$$

Doing this we must take care for contribution of intersected poles.

To calculate thermodynamic limit it is enough to take account poles $0,-1$

$$
e^{-\phi} \simeq\left[1-e^{U+\alpha N \ln \cosh B}\right]^{2^{N}} \simeq \exp \left[-e^{U+N \ln 2+\alpha N \ln \cosh B}\right]
$$

Using formula (24), we derive

$$
<\ln Z>\approx N \ln 2+\alpha N \ln \cosh B
$$

Let us take in to account finite size effect. The saddle point gives

$$
\begin{aligned}
e^{-\phi} & \simeq\left[1-e^{U+\alpha N \ln \cosh B}+\Gamma(E) e^{-E U+\alpha N \ln \cosh (B E)}\right]^{2^{N}} \\
& \simeq\left[e-e^{U+\alpha N \ln \cosh B}+\Gamma(E) e^{-(E+1) U+\alpha N \ln \cosh (B E) \cosh B}\right]^{2^{N}} \\
& =e^{-U+\alpha N \ln \cosh B+N \ln 2+\varphi(U)}
\end{aligned}
$$

So we found connection between $\varphi$ and $U \equiv \ln t$

$$
\ln \varphi=U+U_{0}+\varphi(U)
$$

where

$$
\begin{aligned}
U_{0} & =-(N \ln 2+\alpha N \ln \cosh B) \\
\varphi(U) & =\Gamma(E) \exp [-(E+1) U+\ln \cosh (B E) / \cosh B]
\end{aligned}
$$


Let us express $U$ by $\ln \phi$

$$
u \approx-\ln \phi-U_{0}-\varphi\left(U_{0}\right)
$$

With such accuracy

$$
\begin{aligned}
<\ln Z> & =\int_{0}^{\infty} \ln t\left(e^{\phi} d \phi\right) \\
& =\alpha N \ln \cosh B+N \ln 2+\Gamma(E) e^{N(E+1) \ln \delta+\alpha \ln \left[\cosh (B E)(\cosh B)^{E}\right]}
\end{aligned}
$$

In the last expression we have neglected preexponent besides $\Gamma(E)$. The next subphase appears, when saddle point $E$ coincides with point-2:

$$
\tanh (2 B)=\frac{\alpha \ln \cosh B+\ln 2}{\alpha B}
$$

The equation about subphase of paramagnetic phase demands more careful discussion.

\section{$3 \quad$ Spin-glass phase}

In this case it is enough to intersect only one pole $E=0$

After transformation we have

$$
\begin{gathered}
e^{-\phi}=\left[1+\frac{1}{2 \pi} \int_{-i \infty}^{i \infty+E_{0}} d E \Gamma(E) e^{-E U+\alpha N \ln \cosh (B E)}\right]^{2^{N}} \\
e^{-\phi} \equiv \exp \left[\frac{-\left|\Gamma\left(E_{0}\right)\right|}{h \sqrt{2 \pi}} \exp \left(-E_{0} U+\alpha N \ln \cosh \left(B E_{0}\right)+N \ln 2\right)\right]
\end{gathered}
$$

where $h=\sqrt{\alpha N B}$ and for $E_{0}$ we have equation

$$
\tanh \left(B E_{0}\right)=U / \alpha N B
$$

Let us express again $U$ by $\ln \phi$

$$
\begin{gathered}
\ln \phi \approx E_{0} U+\alpha N \ln \cosh \left(B E_{0}\right)+N \ln 2-\frac{1}{2} \ln N \\
U \approx \frac{1}{E_{0}} \ln \phi+\frac{\alpha N}{E_{0}} \ln \cosh \left(B E_{0}\right)+\frac{N \ln 2}{E_{0}}-\frac{1}{2} \frac{\ln N}{E_{0}}
\end{gathered}
$$


In this formula we deal with expressions like $B E_{0}$ and $U / B$.

$$
\tanh \left(B E_{0}\right)=\frac{\ln \cosh B E_{0}+\ln 2 / \alpha}{B E_{0}}-\frac{\ln N}{2 \alpha N B E_{0}}+\frac{\ln \phi}{\alpha N B E_{0}}
$$

If we neglecting the last terms in (44)then we have

$$
E_{0}=\frac{-B_{c}}{B}
$$

Where at $B_{c}$ disappears entropy. For corrections we have $\left(x=B E_{0}\right)$

$$
\begin{array}{r}
(x \tanh x-\ln \cosh x-\ln 2 \alpha)=-\frac{\ln N}{2 \alpha N} \\
x=x_{0}-\frac{\left(\cosh x_{0}\right)^{2}}{x_{0}} \frac{\ln N}{2 \alpha N} \\
U=\alpha N B \tanh x=\alpha N B \tanh x_{0}-\frac{1}{2} \ln N \frac{B}{B_{c}}
\end{array}
$$

For the free energy we receive

$$
<\ln Z>=\alpha N B \tanh B_{c}-\frac{1}{2} \frac{B}{B_{c}} \ln N
$$

So at phase transition point free energy has jump

$$
-\frac{1}{2} \ln N
$$

as for the case fully connected model

\section{Ferromagnetic Phase}

For $e^{-\phi}$ we have expression

$$
e^{-\phi} \approx G(U) \tilde{G}(U)^{2^{N}-1}
$$

where

$$
\tilde{G}(U)= \begin{cases}1-\frac{\cosh B E_{0}\left|\Gamma\left(E_{0}\right)\right|}{2 \sqrt{\pi}} e^{-E_{0} U+\alpha N \ln \cosh \left(B E_{0}\right)} & , E_{0}<0 \\ \frac{\cosh B E_{0}\left|\Gamma\left(E_{0}\right)\right|}{2 \sqrt{\pi}} e^{-E_{0} U+\alpha N \ln \cosh \left(B E_{0}\right)} & , E_{0}>0\end{cases}
$$

and for $E_{0}$ we have equation

$$
\tanh \left(B E_{0}\right)=\frac{U}{\alpha N B}
$$


Similarly for $G(U)$

$$
\begin{aligned}
& G(U)= \\
& = \begin{cases}1-\frac{\left(\cosh \left(B E_{1}\right)-m \sinh \left(B E_{1}\right)\right)}{2 \sqrt{\pi} \sqrt{1-m^{2}}}\left|\Gamma\left(E_{1}\right)\right| e^{-E_{1} U+\alpha N \ln \left[\cosh \left(B E_{1}\right)-m \sinh \left(B E_{1}\right)\right]} & , E_{1}<0 \\
\frac{\cosh B E_{1}-m \sinh B E_{1}}{2 \sqrt{\pi} \sqrt{1-m^{2}}}\left|\Gamma\left(E_{1}\right)\right| e^{-E_{1} U+\alpha N \ln \left[\cosh \left(B E_{1}\right)-m \sinh \left(B E_{1}\right)\right]} & , E_{1}>0 .\end{cases}
\end{aligned}
$$

and for $E_{1}$

$$
\frac{m \tanh B E_{1}-m}{1-m \tanh E_{1}}=x, \quad x=\frac{U}{\alpha N B}
$$

Let us define function

$$
\begin{aligned}
& f(x)=\frac{1+x}{2} \ln \frac{1+x}{1+m}+\frac{1-x}{2} \ln \frac{1-x}{1+m} \\
& g(x)=\frac{1+x}{2} \ln \frac{1+x}{2}+\frac{1-x}{2} \ln \frac{1-x}{2}+\ln 2
\end{aligned}
$$

Simple calculations give

$$
\begin{aligned}
& -E_{0} U+\alpha N \ln \cosh \left(B E_{0}\right)=-\alpha N g(x) \\
& -E_{1} U+\alpha N \ln \cosh \left(B E_{1}\right)=-\alpha N f(x)
\end{aligned}
$$

We wont to calculate corrections

$$
<\ln Z>-\alpha N B m=\int G(U)\left[1-\tilde{G}(U)^{2^{N}-1}\right] \equiv I
$$

It is convenient to break integration interval

$$
I=\int_{-\infty}^{-U_{0}}+\int_{-U_{1}}^{-U_{1}}+\int_{-U_{1}}^{\infty}
$$

Where

$$
U_{0}=\alpha N B m
$$

and

$$
U_{1}=\alpha N B x_{1}
$$


The value of $x_{1}$ defined from equation

$$
\alpha g x_{1}=\ln 2
$$

In the first integral we see the condition of dominance of ferromagnetic free energy under spin-glass expression:

$$
\alpha g(m)>\ln 2
$$

The main contribution to the finite size correction comes from second integral.

We have two subphases. In the first out to saddle point is in the integral of integration, in the second one-out side.

$$
I \sim \exp \left[-\alpha N f\left(-x_{1}\right)\right], \quad x_{1}>x_{2}
$$

and

$$
I \sim \exp \left[-\alpha N f\left(-x_{2}\right)-\alpha N g\left(x_{2}\right)+N \ln 2\right], \quad x_{1}>x_{2}
$$

where for $x_{2}$ we have

$$
x_{2}=\frac{1-\sqrt{1-m^{2}}}{m}
$$

Our expression (64), (65) coincide with known results of information theory

\section{Summary}

We have calculated finite size effects for Derrida model with weak connectivity.

In the spin-glass phase we recived logarithmic corrections, in paramagnetic-expotential.

In ferromagnetic phase at $T=0$ our results coincide with information theory (the results of random coding). That codes give for the value of connectivity near the critical extremal possible suppression of decoding error probability. For strong connectivity the result is unknown for optimal coding.

Unfortunally known boundaries from information theory show, that Derridas model does not give extremal suppression for the case or high values of connectivity (weak velocity). To clarify situation, we need consider finite $P$ corrections. 
In the work [10, [1] has suggested to consider

$$
<\left|\sigma_{i}\left(\tau, T_{N}\right)\right|>_{\tau}
$$

instead of magnetization at $T=0$, where $T_{N}=\frac{1}{2} \ln \frac{1+m}{1-m}$

Neglecting finite $P$ corrections we received the same result for the exponent, as for the case $T=0$

We would like to thank Yev.Harutunyan for consultations during the work.

We are also grateful L. Basalyga, R.Dobrushin, B.Derrida, Sh.Rouhani. N.Sourlas for useful remarks.

This work was supported by NATO linkage grant LG 9303057, ISF grant MVMOOO and German ministry of research and technology grant 211-5231. D.B. Saakian thanks Ivan Kostov for hospitality in Saclay.

\section{References}

[1] B.Derrida,Phys.Rev.Lett.45(1980),79.

[2] D.Gross, M.Mezard,Nuci.Phys.,B240(1984) 43.

[3] N.Sourlas, Nature 339,(1989),693

[4] D. B.Saakian, JETP Lett.(1992),56, n.5

[5] D. B.Saakian, Phys.Lett.(1993),A180,169

[6] I.Chisar, J.Korner, Information theory,Moscow,(1985)

[7] D. B.Saakian, JETP Lett.(1995),56 n.5.

[8] D. B.Saakian, Sh.Rouhani, .Mod.Phys.Lett. B.(1995)

[9] C.Dominicis, P.Mottishaw, J.Phys.(1987),A20, 1267.

[10] P.Rujan, Phys.Rev.Lett.(1993),70 2961 
[11] H.Nishimori,Phys.,(1994),A204, 1. 


\section{Appendix A}

In this appendix we show, how transform our results for the case of full connectivity and derive results of [1].

For this purposes we introduce coupling $J>0$, so we consider

$$
\begin{aligned}
P\left(j_{i_{1} \ldots i_{P}}\right) & =\left(1-\frac{\alpha N}{2 C_{N}^{P}}\right) \delta\left(j_{i_{1} \ldots i_{p}}\right) \\
& +\frac{\alpha N}{2 C_{N}^{p}}\left[\frac{1+m}{2} \delta\left(j_{i_{1} \ldots i_{p}}-J\right)+\frac{1-m}{2} \delta\left(j_{i_{1} \ldots i_{p}}+J\right)\right]
\end{aligned}
$$

For this purposes it is just enough to replace $B \rightarrow B J, B_{c} \rightarrow B_{c} J$

It is easy to cheek [6], that transition to full connectivity means

$$
J \rightarrow J \alpha^{-1 / 2}, \quad \alpha \rightarrow \infty
$$

For us it means

$$
e^{-\phi}=\left[\int_{-i \infty}^{i \infty} \frac{d E}{2 \pi} \Gamma(E) e^{-E u+\frac{N(J B E)^{2}}{4}}\right]^{2^{N}}
$$

We consider the case $m=0$.

Different expressions for $e^{-\phi}$ depends on the poles, intersected by our loop for saddle

point intergation at $E=2 U / \lambda^{2}$ and the last depends on the temperature. It is easy to derive (A.3) directly. 